\title{
Tratamento cirúrgico da ectopia cordis: relato de três casos e revisão da literatura
}

Arlindo A. RISO*, Miguel BARBARO-MARCIAL*, Orlando LUDOVICI*, Deipara M. A. MACHADO*, Munir EBAID*, José Otávio C. AULER JÚNIOR, Geraldo VERGINELLI*, Adib D. JATENE*

RISO, A. A.; BARBERO-MARCIAL, M.; LUDOVICI, O.; MACHADO, D. M. A.; EBAID, M.; AULER JÚNIOR, J. O. C.; VERGINELLI, G.; JATENE, A. D. - Tratamento cirúrgico da ectopia cordis: relato de três casos e revisão da literatura. Rev. Bras. Cir. Cardiovasc., 6(1): 30-37, 1991.

RESUMO: Entre 1985 e 1990, três neonatos portadores de ectopia cordis (EC) foram admitidos no InCor - FMUSP; dois com defeito do tipo tóraco-abdominal e um do tipo torácico. Todas as crianças tinham anomalias cardiacas associadas: atresia tricúspide tipo I-A, ausência de artérias pulmonares centrais e colaterais sistêmico-pulmonares (um paciente); comunicação interventricular (CIV) e comunicação interatrial (CIA) (um paciente); CIA (um paciente). Uma criança não foi operada, devido a infecção e laceraçāo da artéria pulmonar, indo a óbito por sangramento. As duas outras foram operadas, na tentativa de se recobrir o coração com pele; uma foi a óbito no 1 : dia de pós-operatório, por baixo débito (BD); a outra teve evoluçâo mais longa, indo a óbito no 141 : dia de pós-operatório, causado por insuficiência respiratória. A longa evolução desse último paciente deveu-se a um defeito cardíaco de pouca repercussāo hemodinâmica (CIA) e à colaboraçāo de um cirurgião plástico na equipe cirúrgica. Este trabalho relata esses três casos e faz uma suscinta revisão da literatura.

DESCRITORES: ectopia cordis, cirurgia.

\section{INTRODUÇÃO}

Ectopia cordis (EC) é uma entidade rara, geralmente associada a defeitos cardíacos e de alta mortalidade. O recobrimento do coração deve ser feito ainda no período neonatal, o que torna mais difícil o sucesso cirúrgico. Com o diagnóstico pré-natal e as várias técnicas relatadas na literatura, é possível planejar, mais adequadamente, o tratamento cirúrgico. O objetivo deste trabalho é relatar três casos do Serviço e fazer uma sucinta revisão da literatura.

\section{CASUÍSTICA E MÉTODOS}

\section{Pacientes}

Caso 1: D. B. S., paciente do sexo feminino, nascida de parto normal, a termo, foi internada no InCor -
FMUSP aos 12 dias de vida. Não foi possível a obtenção de outras informações a respeito da gravidez e condiçōes do parto, pois o nascimento ocorreu em outro Estado e nenhum parente acompanhou a criança. Ao exame físico de entrada, a criança apresentava-se com peso de $3550 \mathrm{~g}$ e já se notava um defeito da parede torácica, com o coração totalmente exteriorizado na superfície do tórax. O ápice apontava para a frente e para cima e já havia sinais de infecção na parede torácica próximo ao defeito. Notava-se, também, presença de dois ventrículos, duas aurículas e porção inicial dos grandes vasos da base que emergiam da massa ventricular (Figura 1). À palpação, notava-se um defeito parcial do esterno na sua porção superior. No encaminhamento médico, havia referência de um sangramento espontâneo próximo ao tronco pulmonar, ocorrido no 12 . dia de vida, que tinha sido corrigido em outro Serviço. Nesse mesmo dia, após

Trabalho realizado no Instituto do Coração do Hospital das Clínicas da Faculdade de Medicina da Universidade de São Paulo. São Paulo, SP. Brasil.

Apresentado ao 18: Congresso Nacional de Cirurgia Cardiaca. Rio de Janeiro, RJ, 5 e 6 de abril, 1991.

- Do Instituto do Coração do Hospital das Clínicas da Faculdade de Medicina da Universidade de Sāo Paulo.

Endereço para separatas: Arlindo Riso. Av. Dr. Enéas de Carvalho Aguiar, 44. Divisão Cirúrgica. 05403 São Paulo, SP, Brasil 
RISO, A. A.; BARBERO-MARCIAL, M.; LUDOVICI, O.; MACHADO, D. M. A.; EBAID, M.; AULER JÚNIOR, J. O. C.; VERGINELLI, G.; JATENE, A. D. - Tratamento cirúrgico da ectopia cordis: relato de três casos e revisão da literatura. Rev. Bras. Cir. Cardiovasc., 6(1): 30-37, 1991

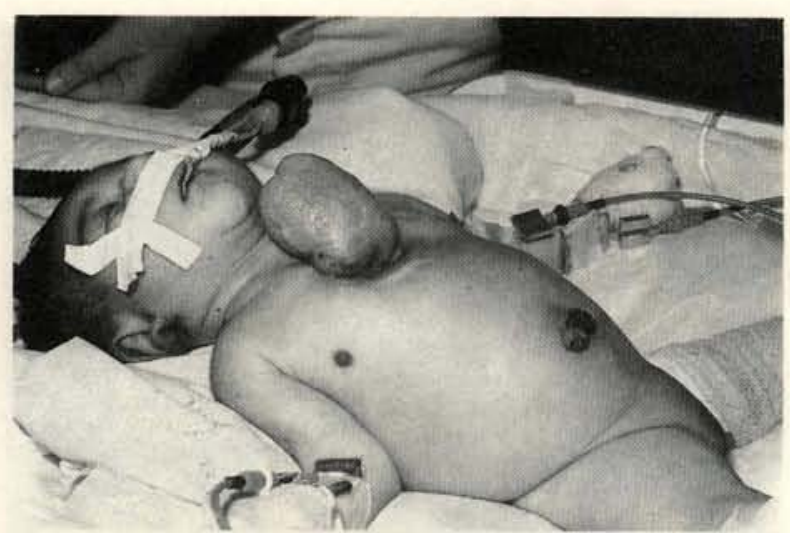

Fig. 1 - Caso 1: ectopia cordis tipo torácico. Nota-se o coração exterio. rizado na porçăo superior do esterno, com ápice para a frente e para cima.

a chegada ao nosso hospital, apresentou novo sangramento no mesmo local, resolvido na própria UTI, sem muita repercussão hemodinâmica. Com objetivo de se diagnosticarem possíveis defeitos cardiacos, a criança foi submetida a cateterismo cardiaco que revelou a presença de uma CIV, uma CIA e canal arterial. No $13^{\circ}$. dia de vida, apresentou novo sangramento, desta vez com grande alteração hemodinâmica, necessitando, além da hemostasia e reposição volêmica, massagem cardiaca e drogas vasoconstritoras. A partir daí, a deterioração clínica foi rápida, com insuficiência renal e diálise peritoneal, apresentando parada cardíaca no 14: e 15 : dias de vida, esta última sem sucesso às manobras de reanimação. A necropsia confirmou presença do defeito parcial do esterno, da CIA tipo forame oval e da CIV $(5 \mathrm{~mm})$ tipo perimembranosa da porção trabecular. Havia, também, um pequeno desvio anterior ao septo interventricular, com calvagamento.da aorta de $20 \%$, porém sem estenose pulmonar. Além disso, não havia tronco braquiocefálico e a artéria subclávia direita era retroesofágica.

Caso 2: P. S., masculino, nascido de parto cesárea, com 38 semanas de gestação, indicada por toxemia gravídica (mãe de 15 anos), com peso $2930 \mathrm{~g}$ e Apgar 8-9, foi transferido ao InCor - FMUSP 10 horas após o nascimento. Nota-se, além da cianose $+1++$, defeito da parede torácica e abdominal, defeito parcial do esterno na porção inferior, coração e um dos vasos da base totalmente exteriorizados e ausência de pele desde o coração até a cicatriz umbilical, onde apenas uma membrana recobria os órgãos abdominais. O coração tinha isomerismo direito e o ápice apontava para a direita; da massa ventricular, apenas um grande vaso emergia e a identificação de dois ventrículos era difícil. $O$ estudo ecocardiográfico diagnosticou atresia tricúspide (AT) com atresia pulmonar e concordância ventrículo-arterial (tipo IA). No primeiro dia de vida, foi submetido a operação com objetivo de se recobrir o coração com pele.
Para tanto, foi feita uma ampla dissecção da pele, liberados os átrios e veias cavas (havia também cava esquerda), as veias pulmonares e a aorta. Como não se conseguiu retalo de pele suficiente, sem prejuízo hemodinâmico, foi necessária a abertura da pleura direta, ressecção de quatro costelas direitas até a linha hemiclavicular e a plicatura do diafragma, para que o coração se acomodasse no tórax. Após 20 minutos do fechamento da pele, houve hipotensão seguida de parada cardiaca. A pele foi prontamente aberta e o coração recuperado após massagem e drogas em altas doses. O coração foi, então, recoberto com retalho de pericárdio bovino suturado nas bordas de pele do defeito. O paciente foi levado à sala de pós-operatório em condições clínicas instáveis (PAM de $40 \mathrm{mmHg}$ e altas doses de vasoconstritores), tendo-se acrescentado prostaglandina, dada à piora da cianose. A criança deteriorou-se rapidamente, devido a baixo débito e acidose de repetiçao, indo a óbito no 1: dia de pós-operatório. A necropsia demonstrou tratarse de cardiopatia congênita complexa caracterizada por: AT-IA, CIA nāo restritiva, ausência de artérias pulmonares centrais e presença de colaterais sistêmico-pulmonares da aorta descendente. Outros achados, além da agenesia dos $2 / 3$ inferiores do esterno, foram sinais indiretos da presença de válvula de uretra posterior.

Caso 3: A. P. S., masculino, nascido de parto cesárea com $3180 \mathrm{~g}$ bem deprimido, foi internado no InCor - FMUSP com um dia de vida. A criança encontrava-se em bom estado geral e o exame físico demonstrou defeito da parede torácica e abdominal, com o coração exteriorizado através do terço inferior do esterno e, como no caso de n: 2, não havia pele desde o coração até a cicatriz umbilical (Figura 2). O estudo ecocardiográfico demonstrou apenas uma CIA tipo FO como defeito cardíaco. A criança foi operada no $7^{\circ}$. dia de vida e a equipe cirúrgica foi formada por um cirurgião cardiovascular e um cirurgião plástico. Feita a liberação de todo o coração, vasos de bases, veias cavas e pulmonares e de toda a pele para recobrir o coração. Não houve prejuízo hemodinâmico, porém havia muita tensão na sutura e isquemia das bordas. Para contornar esse problema, um retralho de músculo retro-abdominal direito foi rodado, deixando-se sua inserção superior com pedículo vascular, e colocado entre o coração e a pele, a fim de dar suporte para uma cicatrização adequada (Figura 3 ). A evolução hemodinâmica decorreu sem nenhuma intercorrência e sem uso de drogas. Foi introduzida nutrição parenteral prolongada, dada a provável entubação endotraqueal por longo período. Após extubação acidental no 7 : dia de pós-operatório, houve tentativa de se manter o paciente com ventilação espontânea, porém sem sucesso. Foi reentubada no 8 : dia de pós-operatório, permanecendo por mais 40 dias (até o $48^{\circ}$ : dia de pós-operatório), após o que foi extubada e assim mantendo-se por 49 dias (até o 97: dia de pós-operatório). Devido a grande quantidade de secreção pulmonar e atelectasias repetidas causadas por infecçāo, necessitou nova entubaçāo 
RISO, A. A.; BARBERO-MARCIAi., M.; LUDOVICI, O.; MACHADO, D. M. A.; EBAID, M.; AULER JÚNIOR, J. O. C.; VERGINELLI, G.; JATENE, A. D. - Tratamento cirúrgico da ectopia cordis: relato de três casos e revisão da literatura. Rev. Bras. Cir. Cardiovasc., 6(1): 30-37, 1991.

por mais 44 dias, até o óbito que ocorreu no 141: dia de pós-operatório, causado por insuficiência respiratória. Provavelmente, esses problemas foram conseqüências de entubação prolongada. Vale a pena ressaltar que, durante toda a evolução, a criança nunca apresentou alteraçōes hemodinâmicas e/ou problemas de cicatrizaçāo (Figura 4). Todas as dificuldades desse período se originavam de problemas ventilatórios e infecciosos, que cediam facilmente ao tratamento instituído, até três dias antes do óbito, quando se tornaram incontroláveis. A necropsia confirmou a CIA como único defeito cardíaco. Não foi encontrado dano alveolar difuso para se explicar a causa da insuficiência respiratória. A causa do óbito permaneceu desconhecida.

\section{DISCUSSÃO}

Ao se rever a literatura, encontramos uma grande variação na definição de ectopia cordis, o que dificulta o entendimento dessa malformação. A definição dada por Abbott, em 1988, (citado por LECA et alii ${ }^{7}$ ), diz tratarse de um malposicionamento do coração que se encontra fora do tórax, exteriorizado na superfície ou dentro

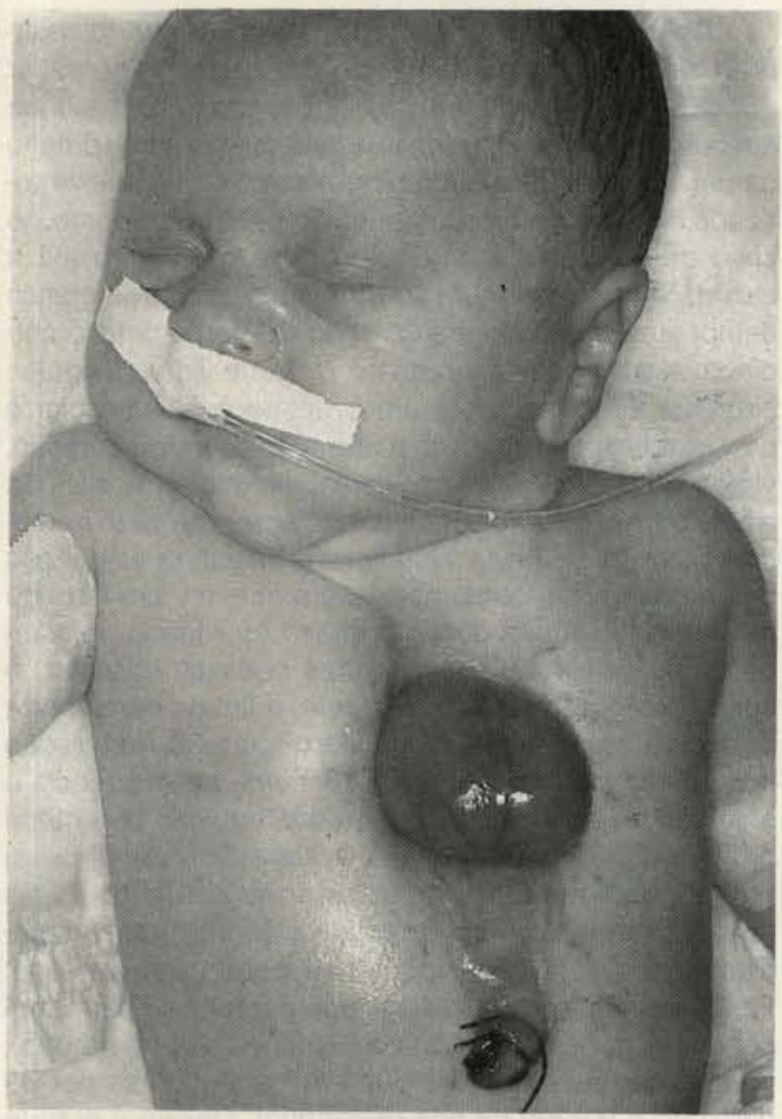

Fig. 2-Caso 3: ectopia cordis tipo toracoabdominal. O coraçāo se exterioriza na porção inferior do esterno: ausẻncia de pele do defeito até a cicatriz umbilical.

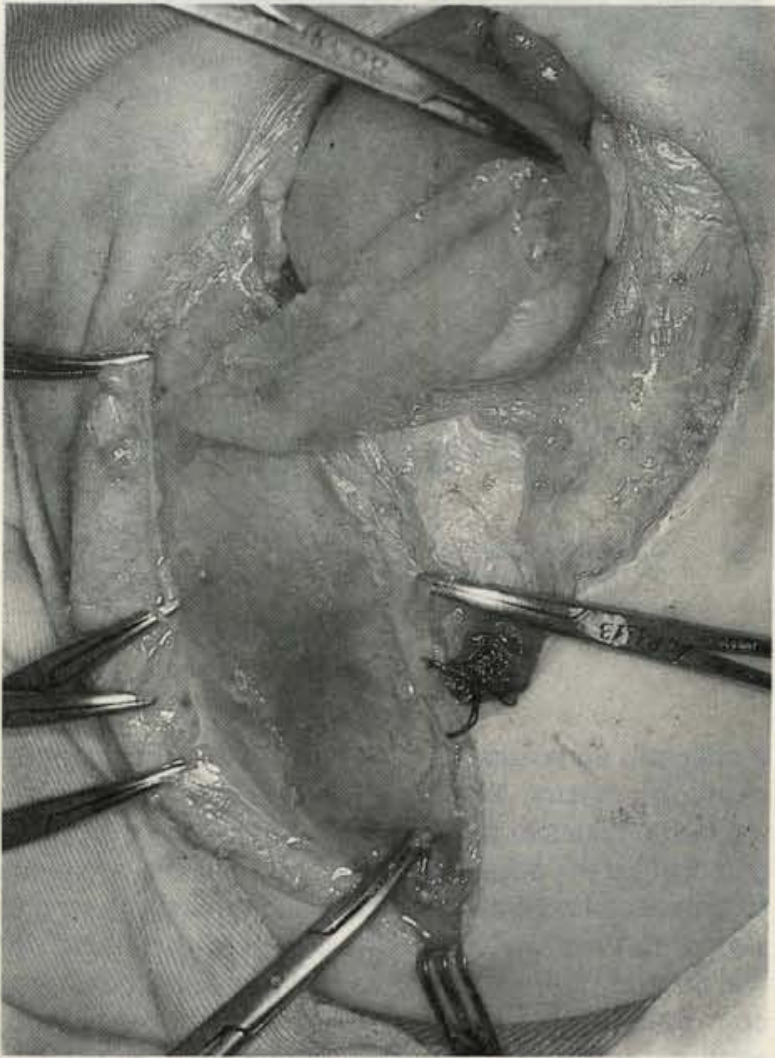

Fig. 3-Caso 3: retalho de músculo retoabdominal direito, com seu pedículo superior rodado sobre o coração.

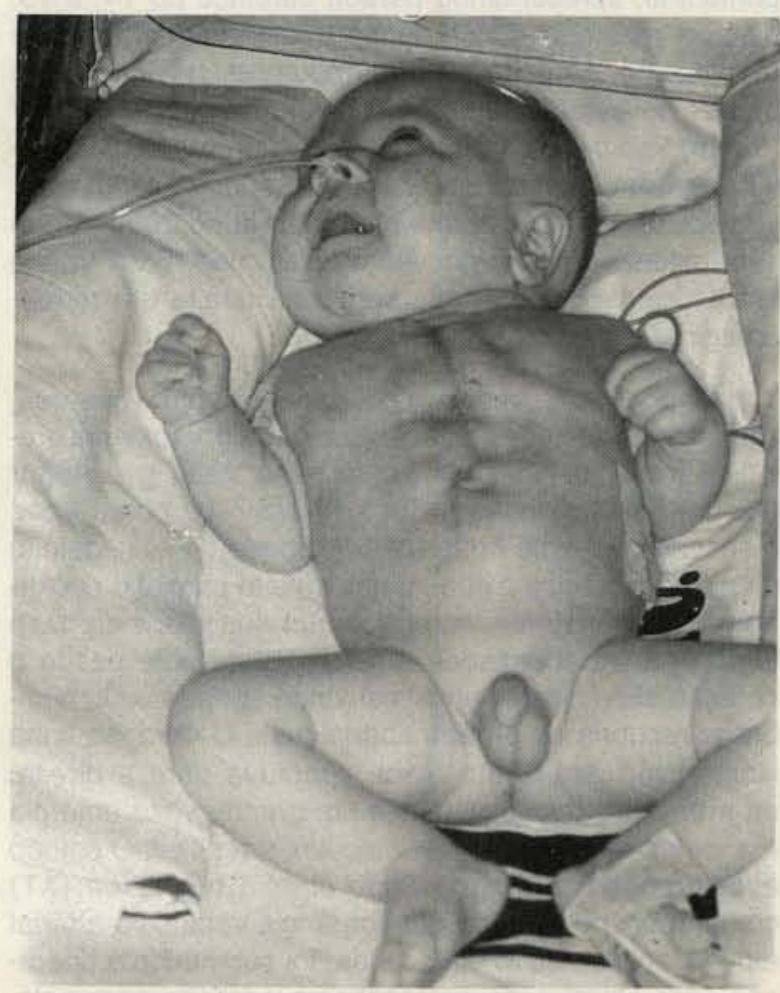

Fig. 4-Caso 3: criança por volta do 60: dia de pós-operatório. 
RISO, A. A.; BARBERO-MARCIAL, M.; LUDOVICI, O.; MACHADO, D. M. A.; EBAID, M.; AULER JÚNIOR, J. O. C.; VERGINELLI, G.; JATENE, A. D. - Tratamento cirúrgico da ectopia cordis: relato de três casos e revisão da literatura. Rev. Bras. Cir. Cardiovasc., 6(1): 30-37, 1991.

TABELA 1

RESUMO DOS CASOS

\begin{tabular}{|c|c|c|c|}
\hline $\begin{array}{l}\text { Detalhes dos } \\
\text { pacientes }\end{array}$ & Caso 1 & Caso 2 & Caso 3 \\
\hline Idade na internação & 12 dias & 10 horas & 1 dia \\
\hline Sexo & $\mathrm{F}$ & M & $M$ \\
\hline Peso de r & - & $2930 \mathrm{~g}$ & $3180 \mathrm{~g}$ \\
\hline $\begin{array}{l}\text { Parto } \\
\text { Tipo de EC }\end{array}$ & $\frac{\text { Normal termo }}{\text { Torácico }}$ & $\begin{array}{c}\text { Cesárea } 38 \text { semanas } \\
\text { Toracoabdominal }\end{array}$ & $\begin{array}{c}\text { Cesárea termo } \\
\text { Toracoabdonimal }\end{array}$ \\
\hline Defeitos cardiacos & $\begin{array}{l}\text { - CIV } \\
\text { - CIA } \\
\text { - Dextroposiçăo de Aorta (20\%) }\end{array}$ & $\begin{array}{l}\text { - AT-IA } \\
\text { - CIA } \\
\text { - Ausência de TP e APs centrais } \\
\text { - Colaterais S-P }\end{array}$ & $-\mathrm{CIA}$ \\
\hline Defeitos não-cardiacos & $\begin{array}{l}\text { - Artéria sublcávia D } \\
\text { retroesofágica } \\
\text { - Ausência de TBC }\end{array}$ & $\begin{array}{l}\text { - Dilatação ureteral } \\
\text { - Hipertrofia vesical }\end{array}$ & 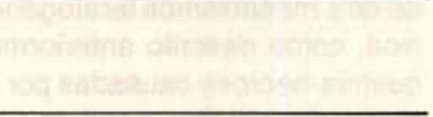 \\
\hline Operaçẵo & 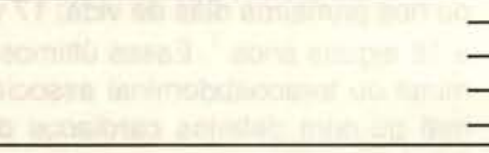 & $\begin{array}{l}\text { - } 1 \text { dia de vida } \\
\text { - Ressecção de costelas } \\
\text { - Plicatura de diafragma } \\
\text { - Cobertura do coração com PB }\end{array}$ & $\begin{array}{l}\text { - } 7 \text { dias de vida } \\
\text { - Cobertura do coração com pele } \\
\text { e músculo retroabdominal }\end{array}$ \\
\hline Evolução & - Sangramento no TP & $\begin{array}{l}\text { - Baixo débito } \\
\text { - Óbito no 1:PO }\end{array}$ & $\begin{array}{l}\text { - Sem alteração hemodinâmica } \\
\text { - Insuficiência respiratória } \\
\text { - Infecção } \\
\text { - Óbito no 141: PO }\end{array}$ \\
\hline
\end{tabular}

$\mathrm{EC}=$ ectopia cordis; $\mathrm{CIV}=$ comunicação interventricular; $\mathrm{CIA}=$ comunicação interatrial; $\mathrm{AO}=$ aorta; $\mathrm{AT}=$ atresia tricúspide; $\mathrm{TP}=$ tronco pulmonar; $\mathrm{APS}=$ artérias pulmonares; $\mathrm{SP}=$ sistêmico-pulmonares; $\mathrm{TBC}=$ tronco braquiocefálico; $\mathrm{PB}=$ pericárdio bovino.

da cavidade abdominal. Uma definição mais abrangente foi proposta por Kanagasuntheram e Verzin, em 1962, (citados por DOBELL et alii ${ }^{1}$ e ULMER et alii ${ }^{11}$ ), em que diz tratar-se de um malposicionamento do coração, parcialmente ou completamente fora do tórax. Com essa definiçāo, fica claramente estabelecido que as fendas de esterno com o coração na cavidade torácica não constituem exemplos de EC. O coração pode estar em várias localizaçōes e o defeito ósseo pode variar desde a ausência completa do esterno, ao esterno totalmente intacto, com o coração na região cervical ou na cavidade abdominal.

A criança descrita por Nils Stensen, em 1671, (citado por POULIAS et alii ${ }^{10}$ e ULMER et alii ${ }^{11}$ ) apresentava ectopia cordis, além do que posteriormente seria chamado de tétrade de Fallot. Talvez essa seja a primeira descrição da doença, embora tenha sido encontrada também em escritos da antiga Babilônia ${ }^{7}$.

Trata-se de anomalia com incidência menor que 1 para 100.000 rascimentos ${ }^{6}$ e raramente encontrada duas vezes pelo mesmo cirurgiāo. A literatura sobre o assunto, portanto, consiste em relato ou revisões de casos acumulados, que nos levam ao entendimento e planejamento cirúrgico adequado.
De acordo com a localização do coração, a EC é classificada em quatro tipos ${ }^{7}$ :

1) Cervical: o coração se exterioriza na porção superior do esterno, localizando-se na região cervical; o esterno pode ter um defeito na sua porção superior ou estar totalmente íntegro.

2) Torácico: através de um defeito ou agenesia do esterno, o coração se exterioriza na superfície torácica.

3) Toracoabdominal: também chamado de síndrome de Cantrell, na qual o coração se exterioriza através de um defeito inferior do esterno, do diafragma e da parede abdominal.

4) Abdominal: o coração se encontra na cavidade abdominal, devido a um defeito do diafragma.

Segundo a revisão feita por LECA et alii ${ }^{7}$, em 1989 , onde foram analisados 219 casos relatados na literatura, a maior incidência entre os tipos de EC foi o torácico $(37 \%)$ e o toracoabdominal ( $36 \%)$, seguidos do abdominal $(11 \%)$ e cervical $(8,5 \%)$. O caso de $n$ : 1 relatado era do tipo torácico e os demais do tipo toracoabdominal, concordando com essa tendência. 
RISO, A. A.; BARBERO-MARCIAL, M.; LUDOVICI, O.; MACHADO, D. M. A.; EBAID, M.; AULER JÚNIOR, J. O. C.; VERGINELLI, G.; JATENE, A. D. - Tratamento cirúrgico da ectopia cordis: relato de três casos e revisâo da literatura. Rev. Bras. Cir. Cardiovasc., 6(1): 30-37, 1991.

É difícil formular-se um conceito embriológico que explique as anomalias de esterno, diafragma e parede abdominal. KAPLAN et alii ${ }^{5}$ observaram a associação de EC com anomalias de rompimento de banda e formulou a hipótese de que o mecanismo teratogênico consiste na compressão mecânica secundária à rotura do córion e/ou saco vitelínico, por volta da 3a semana de gestação. Essa compressão interfere na descida normal do coração, da regiāo cervical ao tórax, comprime o tórax e não permite a fusão do esterno em desenvolvimento. Se isso ocorrer mais tarde, porém antes da $6^{\mathrm{a}}$. semana, quando a fusão do esterno já se completou, a compressāo do tórax pode causar uma fenda no esterno sem EC, já que a descida do coração já teria ocorrido. A EC do tipo toracoabdominal seria resultante da interaçāo de dois mecanismos teratogênicos: a compressão mecânica, como descrito anteriormente, e a compressão-isquemia-necrose causadas por uma banda na região umbilical. Os defeitos cardíacos associados podem representar deformidades secundárias à distorção mecânica do coraçāo em desenvolvimento, seguida do rompimento precoce do córion e/ou saco vitelínico.

Malformações associadas à EC, tanto cardiacas quanto não cardiacas, são muito freqüentes. As não cardiacas foram encontradas em $70 \%$ (45/65) dos casos documentados, sendo a maioria anomalias craniofaciais (hidrocefalia, fenda palatina e fenda labial) ${ }^{7,11}$. Não encontramos tais anomalias em nossos pacientes; o paciente de número 1 tinha ausência de tronco braquiocefálico e artéria subclávia retroesofágica; o paciente de número 2 tinha sinais indiretos da presença de válvula de uretra posterior (dilatação ureteral bilateral e hipertrofia vesical). Dentre os defeitos da parede torácica, a maior ocorrência foi, logicamente, os defeitos do esterno, desde agenesia até defeitos parciais (nossos três casos). Defeitos do pericárdio e do diafragma são também muito freqüentes. Dentre os defeitos da parede abdominal, a onfalocele tem a maior incidência $(60,6 \%$ $40 / 66$ pacientes) ${ }^{7}$. Nenhum dos nossos pacientes apresentou outras anomalias não cardiacas importantes.

Malformações cardiacas associadas à EC foram encontradas em $95 \%$ dos casos bem documentados $(129 / 135)$. Apenas seis pacientes tinham o coração estruturalmente normal, três do tipo torácico e três do toracoabdominal $^{7}$. Os defeitos cardiacos associados são, em regra, hemodinamicamente importantes e necessitam de coraçōes. A CIV, na maioria das vezes associada a estenose pulmonar, estava presente em 60 (46\%) casos, tétrade de Fallot em nove $(6,9 \%)$ e divertículo de ventrículo esquerdo em $12(9,3 \%)$. Outros defeitos também encontrados foram dupla via de saída de ventrículo direito, transposição das grandes artérias, atresia tricúspide, tronco arterioso e outros mais complexos ${ }^{7}$. O caso de número 2 tinha AT-IA com ausência de artérias pulmonares centrais, sendo o fluxo pulmonar proveniente de colaterais sistêmico-pulmonares originárias da aorta descendente; o caso 1 tinha apenas uma CIV e discreta dextroposição de aorta.

Em relação à evolução do feto durante a gravidez, pode-se afirmar que, em geral, 1/3 tem óbito intrauterino, outro $1 / 3$ nasce prematuramente e um último $1 / 3$ tem parto espontâneo a termo ${ }^{11}$. Dos nascidos vivos, a maioria vai a óbito nos primeiros dias de vida ${ }^{7}$. O prognóstico desses pacientes está intimamente relacionado com o tipo de EC e com a presença de defeitos associados, principalmente os cardíacos. O tipo cervical é praticamente incompatível com a vida ${ }^{9}$. O tipo abdominal parece ser o de melhor sobrevida, como ocorreu no caso relatado por Cullerier, em que um soldado se aposentou sem que seu coração interferisse na sua carreira militar (citado por DOBELL et alii ${ }^{1}$ ).

Na literatura, a duraçāo da vida foi relatada em pouco mais de 100 casos: 70 morreram nas primeiras horas, ou nos primeiros dias de vida; 17 viveram alguns meses e 16 alguns anos ${ }^{7}$. Esses últimos tinham EC tipo abdominal ou toracoabdominal associado com coraçāo normal ou com defeitos cardiacos de pouca repercussão hemodinâmica.

Desde 1981, a literatura tem relatado o diagnóstico pré-natal de EC através de ultrassonografia intrauterina ${ }^{11}$. No nosso meio, ESCOBAR et alii ${ }^{2}$ relataram um

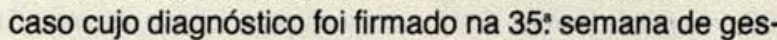
tação. A maioria das indicações para esse estudo foi o esclarecimento de polihidrâmnio. Feito o diagnóstico pré-natal de EC, o objetivo do exame deve ser encontrar, ou não, anomalias cardíacas para se planejar uma conduta, obstétrica ou cirúrgica, precoce e adequada. Caso o diagnóstico seja estabelecido no 1: trimestre, é possível, em alguns países, a interrupção da gravidez. No caso do diagnóstico no último trimestre, deve-se esclarecer bem aos pais a respeito do prognóstico. Tanto num caso como no outro, a interação entre o obstetra. o cardiologista pediátrico, o cirurgião cardiovascular e os pais torna-se extremamente importante.

Várias foram as tentativas de correçāo da EC, porém poucas tiveram sucesso. As complicações curírgicas e/ ou anomalias cardiacas associadas são as maiores causas de óbito. O objetivo inicial do tratamento cirúrgico é o de se providenciar uma cobertura, de pele ou membrana sintética, ao coraçāo exposto ${ }^{1}$. Isso deve ser feito logo após o nascimento, evitando-se, assim, problemas infecciosos, como ocorreu no paciente de número 1 , que deu entrada no nosso Serviço com 12 dias de vida e infecção, provavelmente a causadora de laceração e sangramento do tronco pulmonar.

Muitas técnicas foram utilizadas com o objetivo de acomodar o coração dentro do tórax, ainda no período neonatal. Além da ampla dissecção dos vasos da base e das veias cavas e pulmonares, outras táticas cirúrgicas, como abertura da pleura, ressecção de costelas, plicatura do diafragma e até lobectomia pulmonar já foram 
RISO, A. A.; BARBERO-MARCIAL, M.; LUDOVICI, O.; MACHADO, D. M. A.; EBAID, M.; AULER JÚNIOR, J. O. C.; VERGINELLI, G.; JATENE, A. D. - Tratamento cirúrgico da ectopia cordis: relato de três casos e revisão da literatura. Rev. Bras. Cir. Cardiovasc., 6(1): 30-37, 1991.

realizadas ${ }^{2,7,8}$. Os resultados, porém, não foram satisfatórios por dois fatores: compressão do coração dificultando o enchimento ventricular e/ou angulação dos vasos da base ou veias. A necropsia do paciente de número 2, que em algumas dessas técnicas foram utilizadas, mostrou sinais histológicos compativeis com dificuldade do retorno venoso pulmonar. Mais recentemente, uma membrana sintética tem sido colocada sobre o coração, quando não é possivel recobri-lo com pele ${ }^{1,3,4}$. No paciente de número 2 foi utilizado pericárdio bovino para esse fim, o qual não se mostrou adequado devido ao ressecamento e à deterioração ocorridos em poucas horas de pós-operatório. JONES et alii ${ }^{4}$ citam um caso em que usaram membrana de Sylastic por dois meses e meio, pois os pais mostraram-se contrários à operação devido aos defeitos cardiacos. Embora a criança estivesse estável do ponto de vista cardíaco, uma "úlcera" se formou na pseudomembrana que envolvia o ventrículo. Com o risco de perfuração, foi indicada cirurgia, mas, durante a dissecção, houve sangramento e óbito. Uma técnica mais inovadora foi descrita recentemente por GLASS \& FERNBACH ${ }^{3}$, na qual o uso de expansores de pele, colocados lateralmente ao defeito torácico, permitiu a cobertura do coração com a própria pele. Esse processo todo durou duas semanas e, nesse tempo, o coraçāo ficou protegido por uma membrana sintética.

Após o sucesso de se recobrir o coração com pele, o diagnóstico do defeito cardiaco pode ser feito com mais segurança. Dois problemas, porém, ainda permanecem: a correção do defeito cardíaco e a reconstrução da parede torácica. DOBELL et alii ${ }^{1}$ aconselham que se faça a correção do defeito cardíaco concomitantemente à reconstrução do tórax, se isso for possivel. $\mathrm{O}$ paciente de número 2 era portador de uma malformação cardíaca complexa, que seria de difícil abordagem cirúrgica, mesmo na ausência de EC. Poucos são os relatos de literatura em que houve a correção da parede torácica e do defeito cardiaco, em uma ou duas etapas, com sucesso. Symbas e Ware, em 1973, relataram um caso de $E C$ e divertículo de ventrículo esquerdo, que foi tratado em uma só etapa com o auxilio de circulação extracorpórea (citados por LECA et alii ${ }^{7}$ ). Casos como esse deveriam ser corrigidos simultaneamente na fase neonatal, devido ao risco de rotura espontânea do divertrículo.

A reconstrução da parede torácica depende basicamente do tipo do defeito do tórax. O caso relatado por DOBELL et alii ${ }^{1}$, no qual não havia esterno e nem defeito cardíaco, a reconstrução do tórax foi feita aos 19 meses de vida. Com a ajuda de um cirurgião plástico, foram utilizados cinco segmentos de costelas (de 3,5 a $5 \mathrm{~cm}$ ), retirados a partir da linha hemiclavicular em direção lateral. Colocados paralelamente e transversalmente ao tórax, foram suturados na borda anterior das costelas, de modo a formar uma grade sobre o coraçāo. Após oito meses de evolução, três costelas se ossificaram e as outras ainda apresentam certa maleabilidade. DOBELL et alii ${ }^{1}$ afirmam que o sucesso desse caso deveu-se a dois fatores: 1) ausência de defeito cardíaco; 2) equipe cirúrgica formada por um cirurgião cardiovascular e um cirurgião plástico. A longa evolução (141 dias) e o óbito não relacionado do paciente de número 3 deveram-se, praticamente, aos mesmos fatores. Nesse paciente, o defeito cardíaco era passível de longa evolução (CIA). A atuação do cirurgião plástico também foi de grande importância, por estar mais familiarizado com as técnicas de descolamento e rotação de retalhos.

De acordo com a nossa experiência e da revisão da literatura, salientamos alguns ítens de grande importância no tratamento da ectopia cordis:

1) Providenciar uma cobertura ao coração exteriorizado, com pele ou membrana sintética (temporária), logo após o nascimento.

2) Diagnosticar de modo preciso o defeito cardiaco; a oportunidade da correção desse defeito, concomitante ou não à reconstrução da parede torácica, deve ser avaliado pelo cirurgião.

3) Formar uma equipe cirúrgica contando com a colaboração de um cirurgião plástico.

4) Ter suporte pós-operatório adequado ao tratamento de um neonato em estado grave (intensivista pediátrico, enfermeiras e fisioterapeutas). 
RISO, A. A.; BARBERO-MARCIAL, M.; LUDOVICI, O.; MACHADO, D. M. A.; EBAID, M.; AULER JÚNIOR, J. O. C.; VERGINELLI, G.; JATENE, A. D. - Tratamento cirúrgico da ectopia cordis: relato de três casos e revisão da literatura. Rev. Bras. Cir. Cardiovasc., 6(1): 30-37, 1991.

RBCCV 44205-129

RISO, A. A.; BARBERO-MARCIAL, M.; LUDOVICI, O.; MACHADO, D. M. A.; EBAID, M.; AULER JÚNIOR, J. O. C.; VERGINELLI, G.; JATENE, A. D. - Surgical repair of ectopia cordis: report of three cases and a review of the literature. Rev. Bras. Cir. Cardiovasc., 6(1): 30-37, 1991.

ABSTRACT: Between 1985 and 1990, three neonates with ectopia cordis (EC) were admitted to the InCor - FMUSP; two had thoracoabdominal type and one thoracic type. All patients had associated cardiac anomalies: tricuspid atresia type I-A, abscence of central pulmonary arteries and systemic-pulmonary collaterals (one patient); ventricular septal defect (VSD) and atrial septal defect (ASD) (one patient); ASD (one patient). One child was not operated upon, due to infection in pulmonary artery laceration, with death caused by bleeding. The others were operated upon in order to provide a skin coverage; one died in low cardiac output on the early postoperative period; the other died on the $141^{\text {st }}$ day of postoperative period, caused by respiratory failure. This long evolution was possibly due to a cardiac defect with no significant repercussion (ASD) and to a contribution of a plastic surgeon. This paper describes these three cases and makes a brief review of the literature.

DESCRIPTORS: ectopia cordis, surgery.

\section{REFERÊNCIAS BIBLIOGRÁFICAS}

1 DOBELL, A. R. C.; WILLIAMS, H. B.; LONG, R. W. Staged repair of ectopia cordis. J. Pediat. Surg., 17: 353-358, 1982.

2 ESCOBAR, M.; FRANÇA, N. A. C.; LOBO, F.; MARKMAN FILHO, B.; MAIA, F.; LAGES, E. A.; MARKMAN, P.; BUARQUE, F.; OLIVEIRA, S.; BARBOSA, J. M. M.; LIMA, R. - Ectopia cordis: a respeito de um caso. Rev. Norte-Nordeste Cir. Cardiovasc., 1: 19-22, 1988.

3 GLASS, R. B. \& FERNBACH, S. K. - Ectopia cordis and radiographic changes of a new surgical repair technique: case report. Clin. Radiol., 38: 443-444, 1987.

4 JONES, A. F.; McGRATH, R. L.; EDWARDS, S. M.; LILLY, J. R. - Immediate operation for ectopia cordis. Ann. Thorac. Surg., 28: 484-486, 1979.

5 KAPLAN, L. C.; MATSOUKA, R.; GILBERT, E. F.; OPITZ, J. M.; KURNIT, D. M. - Ectopia cordis and cleft sternum: evidence for mechanical teratogenesis following rupture of the Clorion or Yolk sac. Am. J. Med. Genet., 21: 187-199, 1985.

6 KHOURY, M. J.; CORDERO, J. F.; RASMUSSEN, S. Ectopia cordis, midline defects and chromosome normalities: an epidemiologic perspective. $A m$. J. Med. Genet., 30: 811-817, 1988.

7 LECA, F.; THIBERT, M.; KHOURY, W.; FERMONT, L.; LABORDE, F.; DUMEZ, Y. - Extrathoracic heart (ectopia cordis): report of two cases and review of the literature. Int. J. Cardiol., 22: 221-228, 1989.

8 MILLHOUSE, R. F. \& JOOS, H. A. - Extrathoracic ectopia cordis: report of cases and review of literature. $A m$. Heart. J., 57: 443-444, 1987.

9 MUELLER, G. \& SCHALLER, A. - Ectopia cordis cervicalis: a case report. Tetralogy, 25: 277-281, 1982.
10 POULIAS, G. E.; RAFTOPOULUS, J.; POLEMIS, L.: SKOUTAS, B.; KATSOULI, R. - Total thoracic ectopia cordis with complete abscence of sternum and pericardium and double diverticulum. J. Cardiovasc. Surg., 23: 75-78, 1982.

11 ULMER, H. E.; STOLZ, W.; KUHL, G.; MECHTERSHEIMER, G. - Ectopia Cordis: Bericht ueber einen praenatal diagnotizierten Fall und eine kurze Uebersicht ueber die Literatur. Monatsschr. Kinderheilkd., 137: 468-471, 1989.

\section{Discussão}

\section{DR. LUIZ CARLOS BENTO DE SOUZA São Paulo, SP}

A oportunidade de ter analisado o texto deste trabaIho evidenciou ser o mesmo uma revisão cuidadosa de um tema raramente apresentado. Este comentário tem a finalidade de concordar e dar suporte às conclusões elaboradas, principalmente aquelas relativas à conduta, onde o esquema seqüêncial do tratamento (cobertura do órgão protegendo-o de infecção, diagnóstico e correção da cardiopatia com reconstrução da parede torácica auxiliado por cirurgião plástico) parece ser a a melhor conduta. A nossa experiência resume-se a dois casos, que foram operados no 2 : dia de vida, com ectopia tipo torácica, com ausência de esterno e portadores de cardiopatia. No primeiro caso, tentou-se a cobertura do órgão com pele, porém a distorção e a angulação dos vasos da base foram a causa mortis nas primeiras horas de pós-operatório. No 2 . caso, os sinais infecciosos eram muito evidentes. Resolveu-se recobrir o órgão após dissecção da pele com membrana de pericárdio bovino, colocando-se cateteres para lavagem contínua com solução de povidine (2\%). Faleceu no 2 : dia de pós-ope- 
RISO, A. A.; BARBERO-MARCIAL, M.; LUDOVICI, O.; MACHADO, D. M. A.; EBAID, M.; AULER JÚNIOR, J. O. C.; VERGINELLI, G.; JATENE, A. D. - Tratamento cirúrgico da ectopia cordis: relato de três casos e revisão da literatura. Rev. Bras. Cir. Cardiovasc., 6(1): 30-37, 1991.

ratório, em insuficiência respiratória. Acho que a possibilidade em se conseguir algum resultado deva ser nos casos de ectopia do tipo toracoabdominal, por facilitar mais a reconstrução da parede, e naqueles em que a cardiopatia por mais benigna (CIA, CIV, divertículo do VE). Meus cumprimentos ao Dr. Arlindo e seus colaboradores.

\section{DR. RICARDO DE CARVALHO LIMA Recife, PE}

Inicialmente, gostaríamos de nos congratular com os autores, pela excelente apresentação. Ao nosso ver, o valor deste trabalho encontra-se em trazer para discussão patologia extremamente rara, acontecendo numa freqüência abaixo de 1 por 100.000 nascimentos. $O$ trabalho aqui relatado faz minuciosa descriçāo dos casos apresentados, bem como uma atualizada revisão da literatura. Concordamos com os autores, quanto à dificuldade para se definir "ectopia cordis", sendo a definição dada por Kanagasuntheram e Versin a mais abrangente. A classificação de: cervical, toracocervical, torácica, toracoabdominal (sindrome de Cantrell) e abdominal nos parece bem satisfatória. Dos cinco tipos o mais freqüentes é a torácica, comprometendo $60 \%$ dos casos. A "ectopia cordis" tem sido descrita em outros animais mamiferos e aves. Patten acredita que o defeito se estabeleça em torno da terceira semana de desenvolvimento do feto. Na nossa experiência, no período de julho de 1976 a março de 1990, tivemos a oportunidade de receber no Unitorax - Unidade de Tratamento Cardiotorácico, Real Hospital Português de Beneficência em Pernambuco, duas crianças portadoras de "ectopia cordis". Ambas tiveram o diagnóstico na fase pré-natal através de ultrassonografia de rotina para avaliação da gestação. O caso de número 1 era do tipo torácica $e$, no caso de número 2, foi observada ectopia tipo toracoabdominal. Em ambos os casos, foi possível a colocação do coração dentro do tórax, sendo necessário o uso de material protético em um paciente e, no outro, recobrir o coração com rotação do músculo peitoral direito e esquerdo e aproximação da pele. No primeiro paciente, o pós-operatório foi tumultuado, vindo a falecer na $12^{\mathrm{a}}$. hora de pós-operatório. A necropsia revelou severa cardiopatia associada, coração univentricular tipo ventrículo direito com hipoplasia do arco aórtico. No segundo paciente, as primeiras 48 horas foram bastante animadoras, com o paciente mantendo excelentes condições hemodinâmicas; no entanto, após esse período, o quadro clínico deteriorou devido a sepsis, vindo o paciente a falecer na 80: hora de pós-operatório. Neste caso, o coração era inteiramente normal. Acreditamos que apesar da alta mortalidade desta malformação é possível, com o auxílio do diagnóstico na fase pré-natal através da ultrassonografia, preciso diagnóstico dos defeitos associados, adequada técnica cirúrgica, participação do cirurgião plástico na equipe e extremo cuidado com infecção, obter-se êxito para tão desafiante defeito. Para finalizarmos, gostaríamos de, mai uma vez, parabenizar a equipe de cirurgia cardiaca do Instituto do Coração de São Paulo.

\section{DR. RISO \\ (Encerrando)}

Agradeço os comentários do Dr. Luiz Carlos e, principalmente, a apresentação dos casos por ele acompanhados. Esses exemplos reforçam as dificuldades e os desafios encontrados por aqueles que se propõem a tratar esses pacientes. Ao Dr. Ricardo, gostaria de agradecer pelo enriquecimento a este estudo, através dos comentários sobre a literatura. Concordamos também com os fatores citados que poderão alterar a mortalidade, ressaltando o diagnóstico pré-natal e o auxilio do cirurgião plástico. 